\title{
Efficacy of Percutaneous Sclerotherapy in Low Flow Venous Malformations - A Single Center Series
}

\author{
Saima Ahmad, DMRD \\ Department of Neuroradiology, Lahore General Hospital, Lahore, Pakistan
}

Purpose: We analyzed results of percutaneous sclerotherapy for venous malformations (VMs) in head, neck and extremities.

Materials and Methods: Thirty-five patients with head and neck and extremities VM treated by sclerotherapy with bleomycin and sodium tetradecyl sulphate (STS) were retrospectively reviewed. A pre-treatment magnetic resonance imaging was done for all patients to diagnose the lesion. Each lesion received 1 to 11 sessions (average, 2.7; standard deviation [SD], 2.03). We evaluated percentage reduction in swelling size and a Likert scale review of subjective feelings of the patients.

Results: Sixteen had a complete obliteration; by sclerotherapy alone $(n=13)$ and surgery after a $75 \%$ reduction $(n=3)$. Ten patients had a significant reduction up to $75 \%$ and three patients by $50 \%$. Four had a minimal decrease with reduction of $25 \%$ or less. Follow-up duration of the patients varied from a minimum of 6 months up to 3 years (average, 15.7 months; SD, 7.8 months). Of all patients, three refused further treatment and were lost to follow-up, while another two were referred to a dermatologist. Thirteen patients reported feeling excellent after the sessions. Eight patients claimed to feel slightly better compared to before the sessions started. Only three patients complained of feeling the same before and after the sessions. None of the patients still in follow-up have reported a recurrence of a lesion thus far.

Conclusion: Sclerotherapy using bleomycin and STS as sclerosants is a safe and effective primary treatment for VMs in the head and neck as well as in extremities.

Key Words: Sclerotherapy; Vascular malformations; Magnetic resonance imaging

\section{INTRODUCTION}

The most common type of congenital vascular malformation is venous malformation (VM), having an incidence rate of 2 in 10,000 and a prevalence of 1\%. Often it leads to severe pain and discomfort to patients as well as morbidity and serious local and systematic complications. While the true cause of a VM is still unknown, it is suspected to be a result of abnormal vascular morphogenesis, secondary to heritable or sporadic mutations, altered gene expression, or various environmental factors. $^{2}$

The disease manifests in a variety of symptoms, ranging from no-pulsatile, compressible masses to blue patches on the skin. Forty percent of malformations are found in the head and neck, another $40 \%$ are found in extremities, and the trunk has $20 \%$ of all VMs. VMs are known

\section{Correspondence to: Saima Ahmad, DMRD Department of Neuroradiology, Lahore General Hospital, Lahore, Pakistan \\ Tel: +92-3224122866 \\ Fax: +92-4299264092 \\ E-mail: masterinfluencer@gmail.com}

Received: January 17, 2019

Revised: February 23, 2019

Accepted: February 25, 2019
Copyright $\odot 2019$ Korean Society of Interventional Neuroradiology This is an Open Access article distributed under the terms of the Creative Commons Attribution Non-Commercial License (http://creativecommons.org/licenses/by-nc/3.0) which permits unrestricted non-commercial use, distribution, and reproduction in any medium, provided the original work is properly cited.

pISSN 2093-9043 eISSN 2233-6273 
to grow over time as the patient ages. They often get larger during puberty and pregnancy, and there is never a regression. While most malformations are in the skin or subcutaneous tissues, they often extend into underlying muscle, bone, and even abdominal viscera. ${ }^{3}$

While there is no universally accepted treatment modality, the treatment of choice is percutaneous sclerotherapy alone or coupled with surgical excision for larger lesions. The three primary reasons for pursuing treatment are to alleviate swelling, to reduce troubling symptoms, and for cosmetic reasons. ${ }^{4}$ Bleomycin is an anti-tumor agent known to have a mechanism of action that inhibits the synthesis of Deoxyribose-Nucleic-Acid (DNA), which produces a sclerosing effect on endothelial cells. Due to financial efficacy, easy availability, and minimal side effects, we tried bleomycin as a first line of treatment in head and neck venous malformations. ${ }^{5}$ Sodium tetradecyl sulphate (STS; 3\%) causes maximum endothelial damage with minimal thrombosis leading to fibrosis and shrinking of lesions. ${ }^{6}$ However, swelling, edema, and necrosis are high when STS is used in the head and neck. Similarly, due to its foamy preparation, it is better suited to larger venous malformations in the extremities and trunk?

This study aims to determine the effectiveness of sclerotherapy using different sclerosants for venous malformations in the head and neck and body.

\section{MATERIALS AND METHODS}

\section{Patients}

Our institutional review board approved the study, and consent forms from all patients treated for venous malformations consecutively with bleomycin and STS between July 2015 and February 2018 were received. Once approvals were obtained, the following criteria were applied to select patients: 1) A panel of experts decided on patients to be included in the study. The panel consisted of a plastic surgeon, a general surgeon, and an interventional radiologist; 2) only patients with low-flow lesions were included in the study. Patients with a history of chest infection were excluded. Patients with mixed lesions were also included in the study and were referred to a plastic surgeon after the treatment of a low-flow lesion; 3 ) in order to confirm a diagnosis of lowflow VM, consistent with International Society for the Study of Vascular Anomalies classification for vascular anatomies, a magnetic resonance imaging (MRI) and ultrasound were conducted. The presence of calcifications confirms slowflow lesions on MRI. In cases where there was a suspicion of a high-flow lesion, we performed a catheter angiography under fluoroscopy to rule out a high-flow lesion; 4) after the above radiological confirmations, an additional direct percutaneous injection into the lesion with abnormal venous lakes confirmed the diagnosis; 5) the entire procedure and process for all patients was documented, consent forms were obtained, and images of the malformations were taken; 6) a minimum follow-up of 8 months was established for the study; 7) clinical evaluation of patients was done before every injection and at next visit after 4-6 weeks; 8) a next session was conducted, if needed, after every follow-up; and 9) after completion of sclerotherapy injection and resolution of the lesion, follow-up MRI was done in every case to measure the decrease in lesion size and enhancement pattern.

A number of data-points were selected for review, including patient demographics, location and number of lesions, number of previous unsuccessful surgeries, number of treatment sessions, dose of bleomycin and STS during each session, side effects of treatment, reduction in VM, improvement in patients condition as reported by the patient on a Likert scale, complications, clinical outcome, and duration of follow-ups (Fig. 1). Patient follow-ups were done through clinical assessment, comparison of pre- and post-treatment photographs, and measurement of lesions size based on comparison between the initial and final MRI and degree of contrast enhancement.

In cases where there was a residual lesion without venous flow on percutaneous puncture, meaning that the whole lesion was already sclerosed, the patient was sent to a plastic surgeon, if there was cosmetic disfigurement and it was a large lesion, for the removal of the sclerosed venous malformation.

\section{Procedure}

The sclerotherapy was done using fluoroscopic guidance with local anesthesia and sterile conditions. A 21-24 G butterfly needle was percutaneously injected into the VM until a venous outflow was observed. Phlebography was done using 1-2 $\mathrm{mL}$ of water-soluble iodinated contrast material, confirming needle position within the VM to access venous drainage. Bleomycin/STS was injected into the VM under fluoroscopy. When the injected material entered the malformation, it would replace the previously injected contrast material (ultravist) and push it into the draining vein. The draining 
vein was manually compressed whenever venous drainage approached deep or dangerous intracranial structures.

Up to 10-15 units of bleomycin, 1 unit $/ \mathrm{mL}, 0.5$ unit/Kg, and $0.5-2 \mathrm{~mL}$ of $3 \%$ STS foam preparation were injected in each session. Sessions were scheduled on average 4-6 weeks apart, as is the gold standard for treatment. ${ }^{5,6}$ The main treatment aim was palliation and size reduction of lesion size. Sclerotherapy sessions were repeated until a treating physician was unable to find any further venous pouches to target for treatment. Post-session patients were put on a course of broad-spectrum antibiotics, analgesics, and anti-inflammatory drugs. Photo imaging as well as MRI was done before and after all treatments to objectively gauge size reduction.

\section{Follow-ups and evaluations}

Patient follow-up ranged from 8 months up to a maximum of 30 months. Patient results are presented in Table 1. There was an interventional radiologist and plastic surgeon involved in the ongoing patient follow up. Treating physicians documented outcomes in patients charts using a Likert scale according to the patient's perception response and through a comparison of pre- and post-treatment photographs. The pre- and post-treatment MR images were prospectively reviewed using $\mathrm{T} 1$ and fat-saturated $\mathrm{T} 2$ images. The standard sequences for characterization and diagnosis of suspected vascular malformations included spin echo (SE) or fast SE T1-weighted imaging (T1WI) for regional anatomy, and SE T2-weighted imaging (T2WI) with and without fat saturation to delineate the extent of an abnormality. Gradient recalled echo (GRE) T2* WI was invaluable for differentiating areas of high-flow or hemosiderin deposition from bleeding. ${ }^{8}$

\section{RESULTS}

The series includes a set of data taken from a total of 35 different patients (14 males). The ages of patients varied between 1.5 years and 55 years, and the mean age was 17.5 years with a standard deviation of 11.5 years. Twenty four had head and neck lesions (Fig. 2) and 11 had venous malformations in the trunk and extremities (Fig. 3). Details of lesions involved with the patients can be found in Table 1.

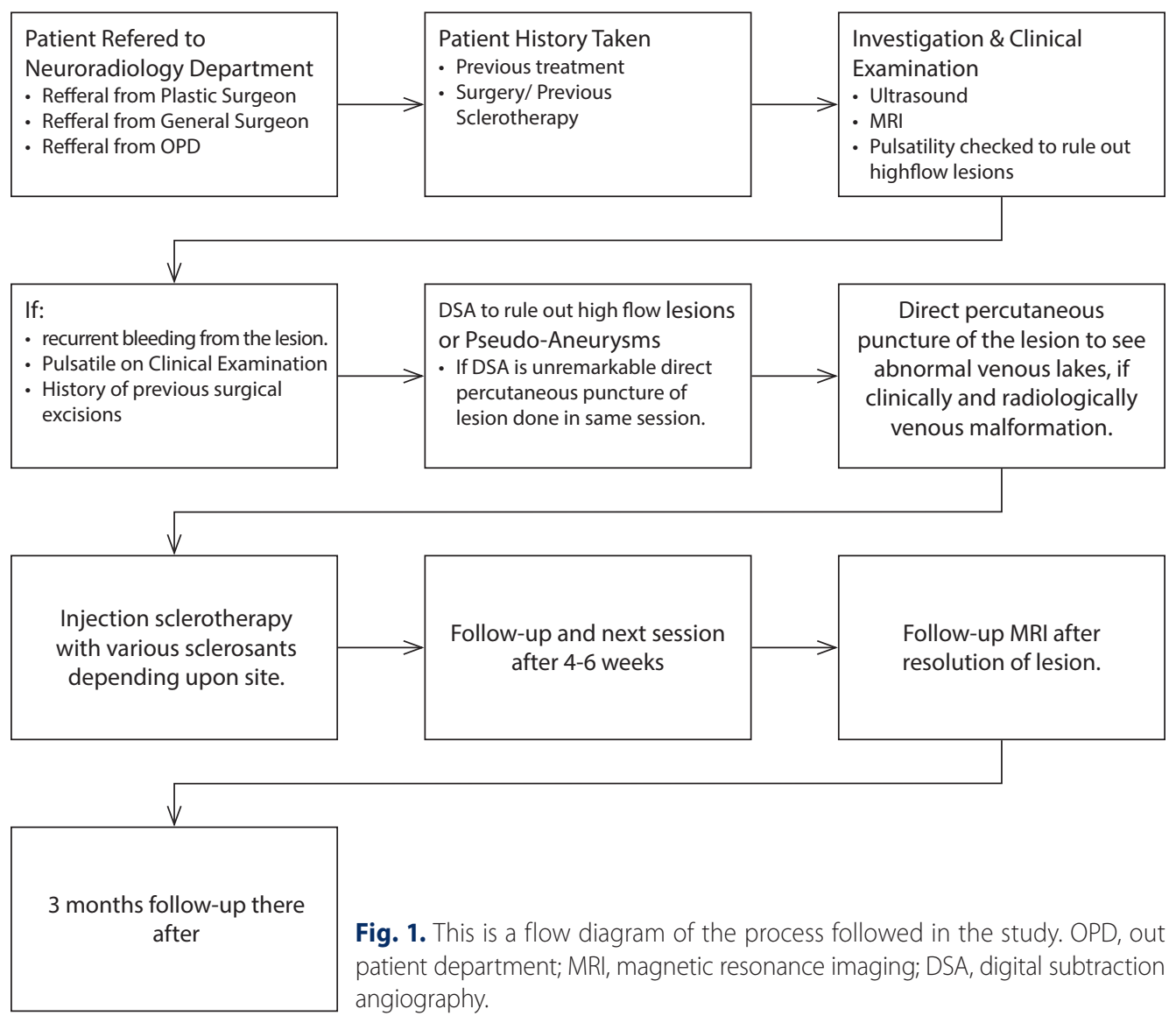




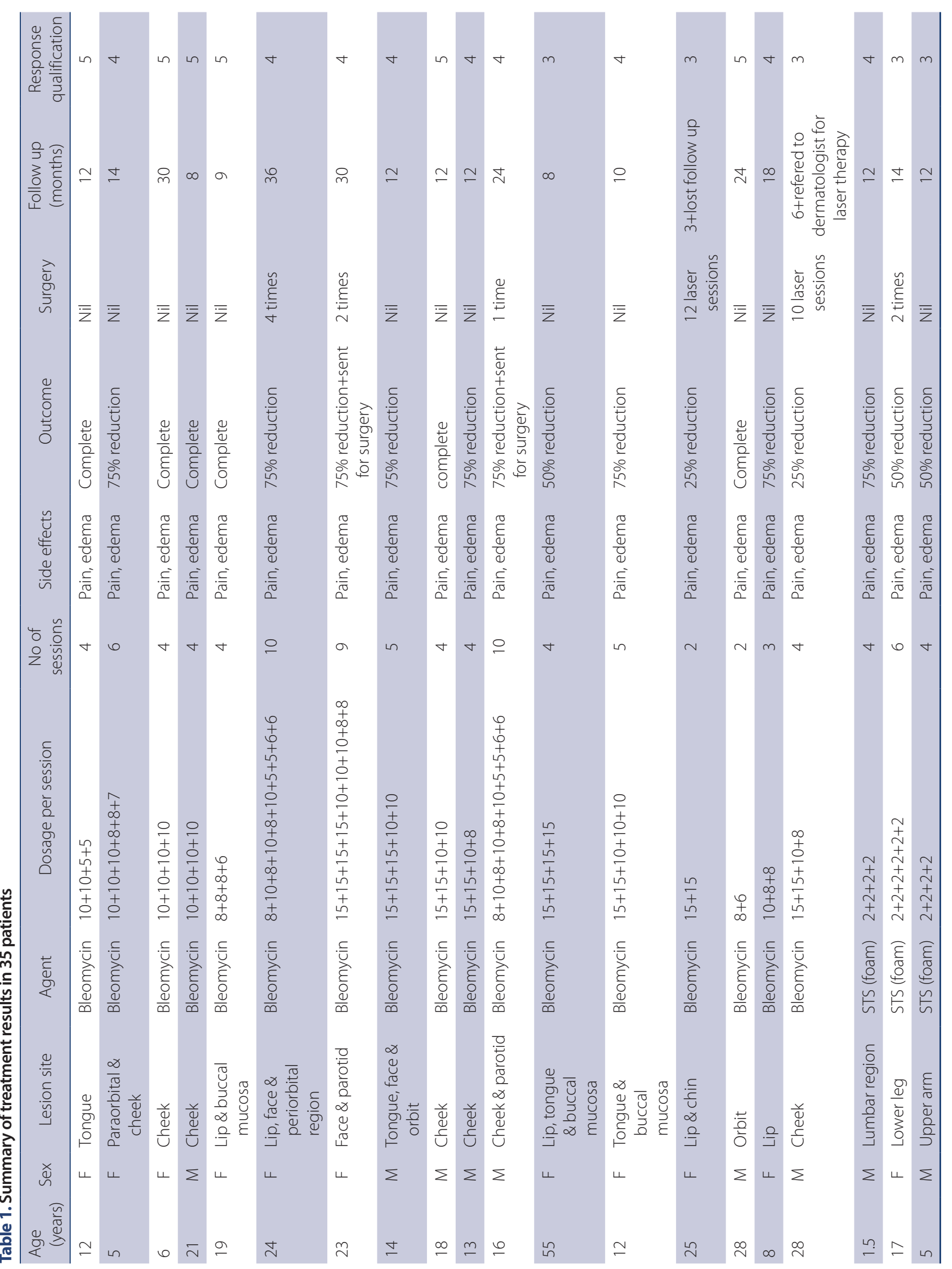




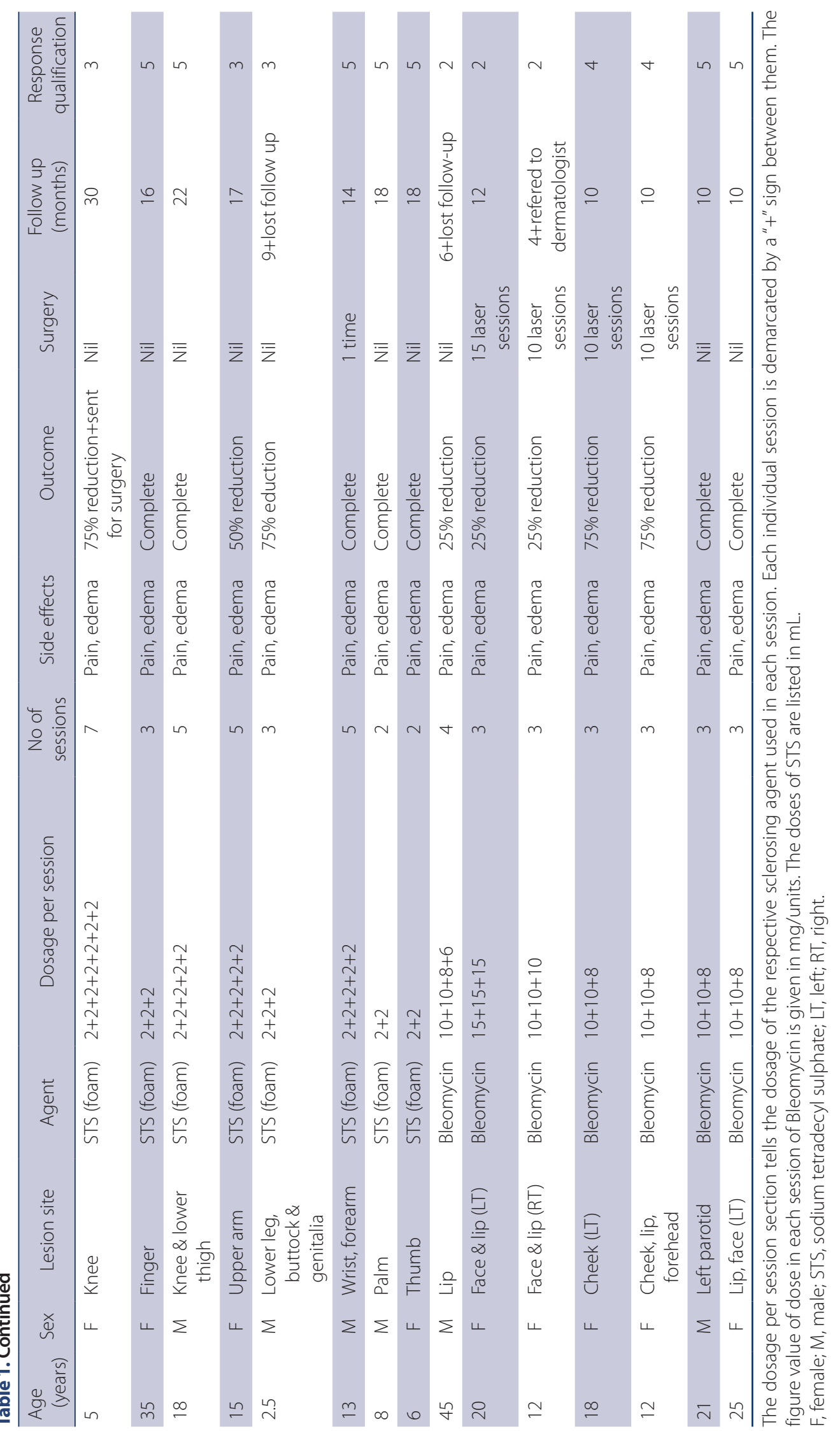


Twelve of the 35 total patients were treated surgically or underwent laser therapy prior to treatment. Both the number of visits and number of injections per lesion varied from patient to patient, with a number of injections ranging from
$1-11$ and the average dose being 10-15 units of bleomycin per injection and a dose of $0.5-2 \mathrm{~mL}$ per STS 3\% foam preparation injection, which was well under the maximum recommended dose of $0.5 \mathrm{~mL} / \mathrm{kg}$.
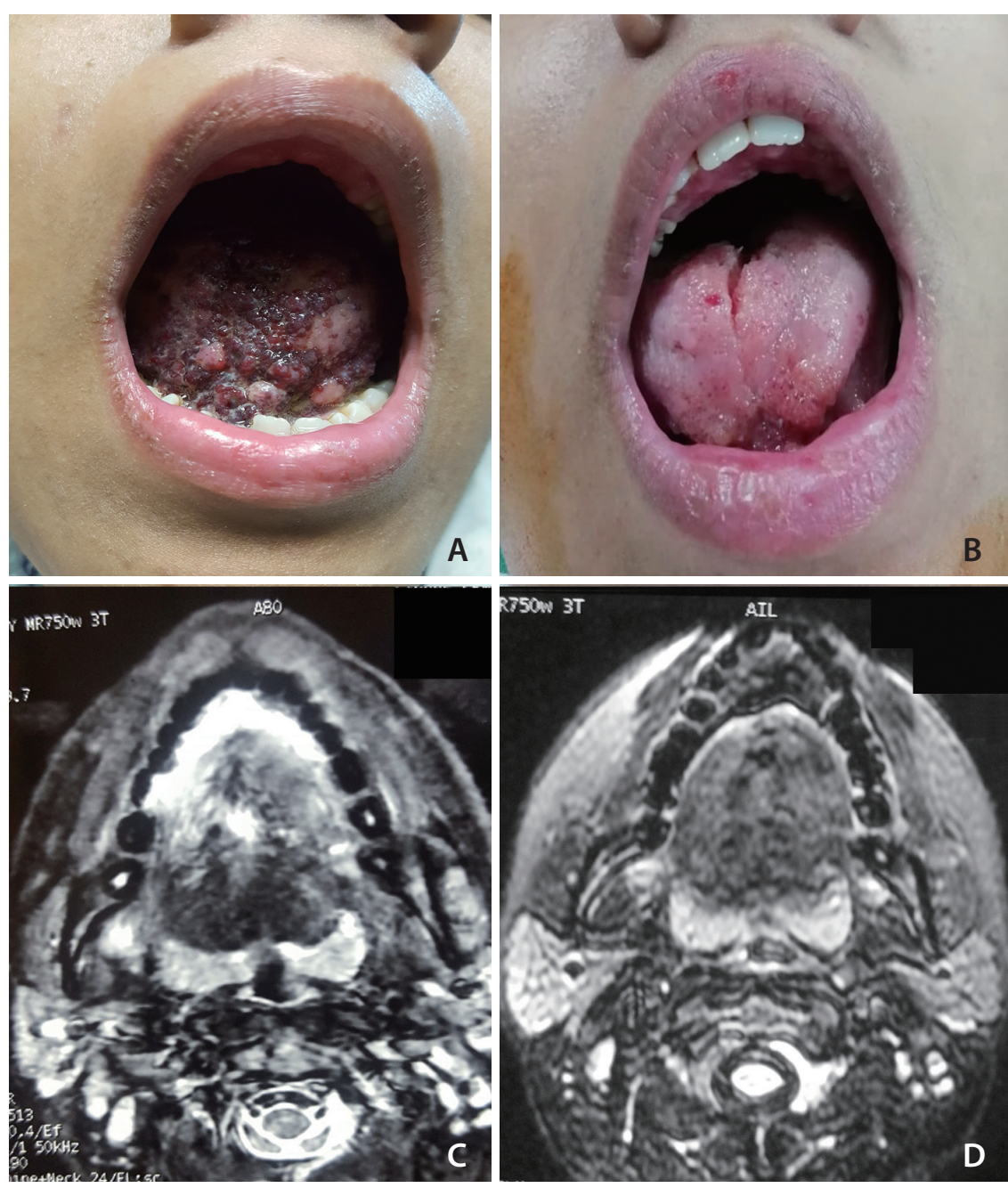

Fig. 2. Twenty-three-year-old female with swelling in the face and lip. Extra-oral view of venous malformation of the oral cavity before treatment (A) after 10 sessions (B). T2-weighted axial magnetic resonance image of an extensive venous malformation of the oral cavity brfore (C) and (D) after treatment.
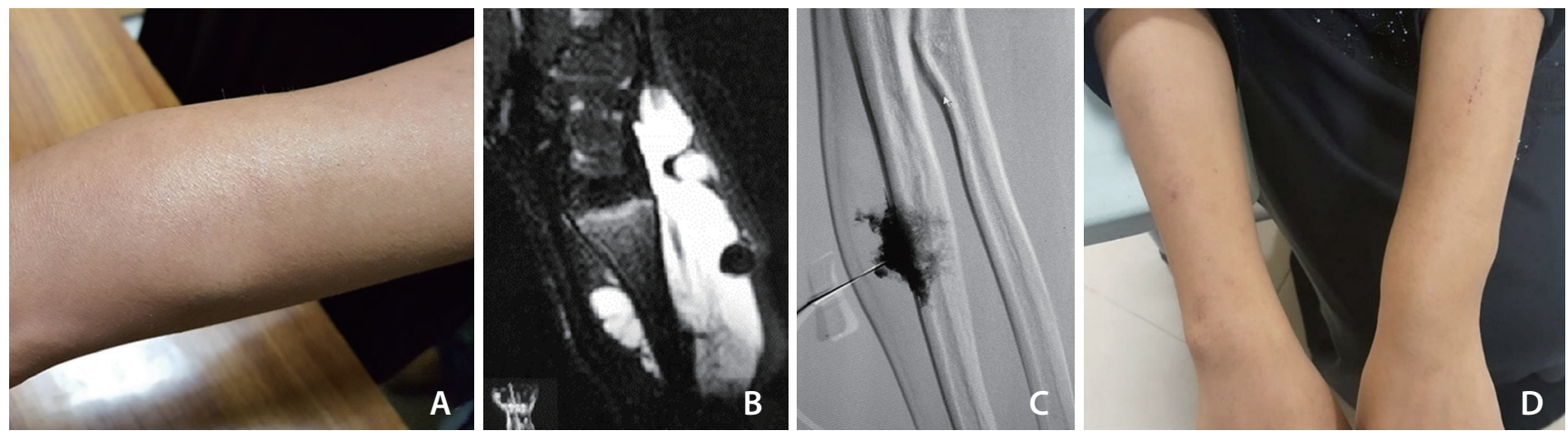

Fig. 3. Sclerotherapy of venous malformation in the forearm (A). T2-weighted magnetic resonance image shows venous malformation with high signal intensities and foci of low signals corresponding to phleboliths (B). Percutaneous phlebography of venous malformation shows irregular venous vascular space (C). Disappearance of the swelling in the left forearm after sodium tetradecyl sulphate (D). 
An average of $0.5 \mathrm{~mL}$ STS for each $2 \mathrm{~mL}$ volume of lesion was used. ${ }^{9}$ Of the 35 patients treated, 16 patients had complete obliteration of the venous malformation; of those 16, 13 were successfully treated with sclerotherapy only, while the other three were referred to surgery after a $75 \%$ reduction in malformation size.

Only four of the patients did not respond well to the therapy and had $25 \%$ reduction in VM size, which was mostly due to the venous malformations being mixed lesions. All patients generally stayed in follow-up, with only three patients lost to follow-up, while another two were referred to the dermatologist for laser therapy once they stopped responding to the sclerotherapy treatment. Laser therapy treatment was for capillary malformations, which cannot benefit from sclerotherapy, and results of laser therapy were not tabulated nor are they included in this study.

There were no signs of hypersensitivity reactions, and the side effects patients faced were pain and edema after injection. Only three of the total group had mild superficial ulceration after STS injection, which healed without scarring. However, no ulceration was observed in cases of bleomycin. All patients were given a 7-day course of anti-inflammatories and analgesics following treatment sessions, while pre-emptive steroids were used in only three cases where the lesion size was extremely large and infected with superficial ulcerations.

\section{DISCUSSION}

Percutaneous sclerotherapy has been established as the first line treatment for $\mathrm{VM}^{10}{ }^{10}$ There are several sclerosing agents, including sodium morrhuate, ethanol amine oleate, ethanol, bleomycin, STS 3\% foam preparation, and hypertonic saline.' Diagnosis is primarily based on clinical presentation and patient history, but a confirmation is usually done with MRI and digital subtraction angiography in select cases. ${ }^{11}$ Venous malformations are lobulated, mass like, infiltrating, tubular lesions due to single or multiple venous lakes of slow flowing blood. VMs have no borders with no regard for other tissue types and can grow and spread across any type of tissue layer. Venous malformations show intermediate signal intensity on $\mathrm{T} 1$ weighted images and hyperintense signals on $\mathrm{T} 2$ and short-TI inversion recovery (STIR) weighted images. A STIR sequence is the most targeted way to detect any involvement and depth of lesion. During a STIR sequence, the lesion is distinctly visible against a hyperintense background of low-signal-intensity fat, muscle, and osseous structures. ${ }^{12}$

STS is a very popular sclerosing agent. Similar to STS, another often used agent is polidocanol. Our center however prefers the use of STS foam preparation over polidocanol due to fewer side-effects and its foamy preparation. The most successful treatment is with STS when applied in a larger VM due to the fact that its sclerosing ability is dependent on blood flow and size of feeding blood vessels.

Another sclerosing agent used in our study is bleomycin. According to the literature, it has been recorded that bleomycin offers great control of symptoms in patients with VMs when used as a sclerosing agent. ${ }^{2}$ It is considered as an effective and safe method of treatment with minimal side effects. The largest concern with bleomycin is the potential development of pulmonary fibrosis, a major complication when using bleomycin as recorded in the treatment of cancer patients. However, there is no recorded case in the literature where pulmonary fibrosis resulted from sclerotherapy. This is because, as was shown by Griffiths et al. ${ }^{13}$, there is a minimal amount of bleomycin in the bloodstream after a sclerotherapy session, as Lonescu found no detectable bleomycin in blood samples in his patients when tested 10 minutes as well as 24 hours after the therapy sessions when treating for hemangioma. ${ }^{2}$

The treatment effectiveness of sclerotherapy has always been evaluated using various measures. These include quantitative measures like patient surveys, Likert scaled results on a number of questions ranging from pain reduction, sense of wellbeing, general health, and mobility, as well as cosmetic self-assessment in females. Another method involves review by clinicians before and after photography and MRI, coupled with clinician documentation charts. A series of 13 patients evaluated after treatment with sodium tetradecyl sulphate of VM in the head and neck by Alakailly et al. ${ }^{10}$ concluded that direct intralesional injection of 3\% STS solution is a simple, safe, and effective therapy for head and neck malformations.

In another study, Muir et al. ${ }^{14}$ reported intralesional use of bleomycin treatment for hemangiomas and congenital vascular malformations. It was a prospective review of 95 patients where it was seen that intralesional bleomycin injection therapy is an effective treatment for VMs having a diverse range of presentations. Our study, while not applied to a diverse range, was limited only to low-flow lesions and showed similar results to that of Muir. While Muir targeted a larger array of malformations, our focus was primarily on low- 
flow malformations in the head and neck.

Finally, another study by Horbach et al. ${ }^{7}$ conducted a systemic review of sclerosing agents and sclerotherapy for lowflow venous malformations in the head and neck. The study found that most sclerosants used pingyanmycin, absolute ethanol, OK 432, ethanolamine oleate, bleomycin, polidocanol, doxycycline, and sodium tetradecyl sulphate. It was found that absolute ethanol led to high complications rates, and all sclerosants were effective with an overall response rate between $71-100 \%$. In the largest case series on the subject, 121 patients, 91\% of whom had peripheral extremities VMs, reported improvement with sclerotherapy alone.? Keeping in mind the work done by Sophie, we did not use ethanol in our patients; we focused only on bleomycin as it promised the safest and most effective possible outcomes. Furthermore, our study established that bleomycin has a very rapid and effective result when treating low-flow lesions.

\section{CONCLUSION}

We found that percutaneous sclerotherapy is an effective method for treating slow-flow venous malformations in the head and neck and body. Bleomycin and STS (3\% foam) are effective sclerosing agents. In this series complete symptomatic relief was observed in both the head and neck and trunk. Given that our data-set was smaller in comparison to other studies, we cannot use it as a definitive guide for treatment guidelines. However, follow-up studies on the subject can be expected to yield more concrete results.

\section{REFERENCES}

1. Behravesh S, Yakes W, Gupta N, Naidu S, Chong BW, Khademhosseini $A$, et al. Venous malformations: clinical diagnosis and treatment. Cardiovasc Diagn Ther 2016;6:557-569

2. Spence J, Krings T, terBrugge KG, da Costa LB, Agid R. Percutaneous sclerotherapy for facial venous malformations: subjective clinical and objective MR imaging follow-up results. AJNR Am J Neuroradio/ 2010;31:955-960

3. Dubois J, Soulez G, Oliva VL, Berthiaume MJ, Lapierre C, Therasse E. Soft-tissue venous malformations in adult patients: im- aging and therapeutic issues. Radiographics 2001;21:1519-1531

4. Rautio R, Laranne J, Kähärä V, Saarinen J, Keski-Nisula L. Long-term results and quality of life after endovascular treatment of venous malformations in the face and neck. Acta Radiol 2004;45:738-745

5. Mathur NN, Rana I, Bothra R, Dhawan R, Kathuria G, Pradhan T. Bleomycin sclerotherapy in congenital lymphatic and vascular malformations of head and neck. Int J Pediatr Otorhinolaryngol 2005;69:75-80

6. Bajpai H, Bajpai S. Comparative analysis of intralesional sclerotherapy with sodium tetradecyl sulfate versus bleomycin in the management of low flow craniofacial soft tissue vascular lesions. J Maxillofac Oral Surg 2012;11:13-20

7. Horbach SE, Lokhorst MM, Saeed P, de Goüyon Matignon de Pontouraude CM, Rothová A, van der Horst CM. Sclerotherapy for low-flow vascular malformations of the head and neck: a systematic review of sclerosing agents. J Plast Reconstr Aesthet Surg 2016;69:295-304

8. Bashir U, Shah S, Jeph S, O'Keeffe M, Khosa F. Magnetic resonance (MR) imaging of vascular malformations. Pol J Radiol 2017;82:731-741

9. Balasundaram I, Al-Hadad I, Rehman K, McCafferty I, Monaghan A. The use of foam sclerotherapy to treat low-flow vascular malformations of the head and neck. J Surg Case Rep 2014;2014:rju095

10. Alakailly X, Kummoona R, Quereshy FA, Baur DA, González AE. The use of sodium tetradecyl sulphate for the treatment of venous malformations of the head and neck. J Maxillofac Oral Surg 2015;14:332-328

11. Konez O, Burrows PE. Magnetic resonance of vascular anomalies. Magn Reson Imaging Clin N Am 2002;10:363-388, vii

12. El-Merhi F, Garg D, Cura M, Ghaith O. Peripheral vascular tumors and vascular malformations: imaging (magnetic resonance imaging and conventional angiography), pathologic correlation and treatment options. Int J Cardiovasc Imaging 2013;29:379393

13. Griffiths $T L$, Burr ML, Campbell IA, Lewis-Jenkins V, Mullins J, Shiels $K$, et al. Results at 1 year of outpatient multidisciplinary pulmonary rehabilitation: a randomised controlled trial. Lancet 2000;355:362368

14. Muir T, Kirsten M, Fourie P, Dippenaar N, Ionescu GO. Intralesional bleomycin injection (IBI) treatment for haemangiomas and congenital vascular malformations. Pediatr Surg Int 2004;19:766773 